\title{
Manejo de la atribución hostil en el aula a partir del currículo educativo especializado'
}

\author{
Ricardo Arrubla Sánchez ${ }^{2}$
}

Recibido: 23-07-2017

Aceptado: 20-09-2017

\section{RESUMEN}

El objetivo de esta investigación es la construcción de un modelo educativo para prevenir el comportamiento disocial y delictivo en adolescentes a partir de la teoría de guiones cognitivos de Bandura, y sustentado desde el uso de la razón jurídica y práctica en adolescentes por medio de la incorporación de la cultura de la legalidad en los colegios. Es un estudio descriptivo explicativo, realizado con dos grupos focales integrado por estudiantes de grado 11 a quienes se les realizó entrevista semiestructurada, y la aplicación de la Escala de Conducta Antisocial y Delictiva, Cuestionario de Agresión Proactiva-Reactiva -RPQ- "Reactive-Proactive Aggression Questionnaire". En la población en estudio se identificaron conductas relacionadas con la impulsividad, agresividad, búsqueda de sensaciones, y narrativas violentas, generando en los estudiantes el síndrome de atribución hostil, y un déficit en el procesamiento de la información. Como conclusión principal, la modificación estructural de la cognición negativa en los estudiantes se debe llevar a cabo a partir de la creación de un neurocurrículo que facilite la mediatización del significado, tanto en el nivel cognitivo como en el nivel afectivo.

\footnotetext{
1. Este artículo es resultado de la investigación: "Estudio pedagógico cualitativo para el diseño de un modelo de manejo de la conducta antisocial juvenil en planteles educativos desde un curso de formación sustentado en la razón jurídica y práctica", desarrollado dentro del marco del proyecto cultura de la legalidad con un grupo de estudiantes de grado once.

2. Doctorante en Ciencias de la Educación. Docente investigador de la Fundación Universitaria del Área Andina. Analista de modelos mentales y conceptuales para el aprendizaje avanzado de conocimientos y la solución de problemas científicos. Correo: rarrubla@areandina.edu.co - jessieconsultores@ hotmail.com ORCID: https://orcid.org/0000-0003-1548-8195

Google Académico: https://scholar.google.es/scholar?hl=es\&as_sdt=0\%2C5\&q=rica rdo+arrubla+sanchez\&oq=ri
} 
Palabras clave: Guiones cognitivos, atribución hostil, neurocurrículo, aprendizaje, resiliencia, plasticidad neuronal.

\title{
Management of hostile attribution in the classroom based on the specialized educational curriculum
}

\begin{abstract}
The objective of this research is the construction of an educational model to prevent behavioral and criminal behavior in adolescents from the theory of cognitive scripts of Bandura, and sustained from the use of legal and practical reason in adolescents through the incorporation of the culture of legality in schools. It is a descriptive - explanatory study, conducted with two focus groups composed of 11 th grade students who underwent a semi - structured interview, and the application of the Antisocial and Criminal Behavior Scale, the Reactive-Reactive Aggression Questionnaire -RPQ"Reactive- Proactive Aggression Questionnaire ". In the study population, behaviors related to impulsivity, aggressiveness, search for sensations, and violent narratives were identified, generating in the students the hostile attribution syndrome, and a deficit in the processing of information. As a main conclusion, the structural modification of negative cognition in students must be carried out from the creation of a neurocurriculum that facilitates the mediatization of meaning, both at the cognitive level and at the affective level.
\end{abstract}

Keywords: Cognitive scripts, hostile attribution, neurocurriculus, learning, resilience, neuronal plasticity.

\section{Gestion de l'attribution hostile en classe sur la base du programme d'enseignement spécialisé}

\begin{abstract}
RÉSUMÉ
L'objectif de cette recherche est la construction d'un modèle éducatif visant à prévenir les comportements et comportements criminels chez les adolescents à partir de la théorie des scripts cognitifs de Bandura, et à
\end{abstract}


partir de l'utilisation de la raison légale et pratique chez les adolescents par I'incorporation de la culture de la légalité dans écoles. II s'agit d'une étude descriptive - explicative, menée auprès de deux groupes de discussion composés d'élèves de $11 \mathrm{e}$ année ayant subi un entretien semi-structuré, et de l'application de l'Échelle des comportements antisociaux et criminels, du questionnaire sur l'agression réactive-réactive -RPQ- Questionnaire sur l'agression ". Dans la population étudiée, des comportements liés à l'impulsivité, à l'agressivité, à la recherche de sensations et à des récits violents ont été identifiés, générant chez les étudiants le syndrome d'attribution hostile et un déficit de traitement de l'information. En conclusion principale, la modification structurelle de la cognition négative chez les étudiants doit être réalisée à partir de la création d'un neurocurriculum facilitant la médiatisation du sens, tant au niveau cognitif qu'affectif.

Des mots clés: plans cognitif, attribution hostile, neurocurriculum, apprentissage, résilience, plasticité neuronale.

\section{Introducción}

Mediante el desarrollo de este artículo se pretende proponer un modelo pedagógico sistémico para el manejo de adolescentes con trastorno disocial. Por lo tanto, la sustentación de la información se realizará según la siguiente estructura: primera parte el planteamiento teórico y conceptual del problema a partir de la teoría del aprendizaje social y de los mecanismos de desconexión moral según Luria y Bandura, posteriormente se presentan los problemas cognitivos identificados en un grupo de jóvenes a partir de la teoría de los guiones $y$, finalmente el manejo que debe darse al adolescente a nivel institucional, desde los contenidos del currículo hasta el trabajo con sus redes de influencia.

La fundamentación argumentativa del artículo propone que el comportamiento agresivo es consecuencia de guiones cognitivos aprendidos durante la infancia, y que son almacenados en la memoria, los cuales se activan en diferentes situaciones sociales. Mientras tales guiones 
no sean modificados, las personas tienden a actuar bajo los parámetros establecidos por su forma de pensar.

Según las investigaciones de Bartlett y Nadel, no sólo los factores individuales sino también culturales, ejercen una influencia sobre los patrones estructuradores o deformantes del recuerdo, recayendo su efecto, en especial sobre las preferencias estéticas y el gusto artístico. Para Bandura (1987) en sus libros (1991) Aprendizaje social y desarrollo de la personalidad (1974), Teoría de la agresión (1963), y Teoría de los mecanismos de desconexión moral (2004), son ocho mecanismos los que inciden en su comportamiento: racionalidad moral, comparación ventajosa, lenguaje eufemístico, minimización, ignorancia o distorsión de las consecuencias, deshumanización, atribución de culpa, desplazamiento de responsabilidad y difusión de responsabilidad.

Por su parte, Crick y Dodge (1994), explican el fenómeno a partir del denominado sesgo de atribución hostil, el cual resulta fundamental, ya que las personas ofrecen explicaciones del comportamiento humano basándose más en la persona que en los factores que influyen en su comportamiento. Por último, aparecen asociadas las teorías propuestas por Huesmann (1988), denominadas guiones cognitivos agresivos, quien sostieneque el aprendizaje de los guiones cognitivos sucede de la misma forma que el aprendizaje intelectual, con un "proceso de codificación de comportamientos observados", finalmente se tienen en cuenta las ideas hechas por Gibbs, Potter y Goldstein (1995), llamadas distorsiones cognitivas auto-sirvientes que presentan a las distorsiones como procesos cognitivos que influyen en la concepción de un hecho o experiencia social. Las investigaciones evidencian que, dichas distorsiones, tienen un nivel de incidencia, según el grado de internalización por parte de los sujetos de las normas y valores morales colectivos.

Al respecto, los estudios adelantados por expertos nos proporcionan evidencias de la influencia que sobre la conducta tienen los sistemas de referencia culturales. Lo que a menudo se considera como una respuesta natural a un estímulo particular puede ser natural, al ser adquirido a través de las normas y modelos sociales que se encargan de su normalización. Por lo tanto, la percepción que el individuo construye está condicionada por sus pasadas experiencias, por las tradiciones, y el sistema de creencias que el grupo también acepta e incorpora dentro de sus prácticas y lógicas de convivencia. 


\section{Procesos cognitivos y aprendizaje}

Por medio del proceso de socialización el individuo interioriza las normas y valores morales de su contexto social, su incorporación se da mediante procesos cognitivos que son en su mayoría implícitos y no cuestionados (Barrantes, Beltrán \& Pérez, 2016). La interiorización progresiva, ejerce un control sobre las actitudes y el comportamiento moral, mediante un sistema de autorregulación que previene la ejecución de la conducta reprobable y estimula las actitudes y el comportamiento aceptable. Autores como Bandera, Baibanelli, Caprara, y Pascore, (1996) establecen la existencia de un sistema de control o autorregulación de actitudes y comportamientos en tres fases: 1) focalizándose en el significado de la propia conducta con relación a los principios socio morales, 2) autoevaluando su propio comportamiento y los efectos que éste tiene sobre sí mismo, tales como el sentimiento de culpa, el auto desprecio de sí mismo o hacía los otros.

Se determina que ante cada decisión moral se activa este sistema de autorregulación del comportamiento en sus tres fases o momentos, ante lo cual el sujeto emitiría conductas y actitudes siempre coherentes (Langer, 2016). Según estas teorías, el comportamiento agresivo y antisocial, es el resultado de guiones negativos que inciden en la interpretación de la información del contexto social, y que producen alteraciones en la percepción y comprensión de un hecho social (Tahull, 2016).

Para Dodge (1993) y Huesmann y Eron (1989), los guiones cognitivos distorsionados se van incorporando a la memoria a partir de las experiencias significativas y las condiciones del contexto, sus ideas sirven de marco para la reacción ante nuevas situaciones sociales, de manera que, si las bases interpretativas se mantienen estables, se pueden profundizar en la memoria, hasta llegar a ser un pensamiento automático, o perder fuerza y dar paso a otra lógica de interpretación. Siguiendo la conceptualización de Dodge (1993) existen diferentes factores que inciden en la forma como las personas adquieren referentes cognitivos distorsionados o negativos, entre los que están: déficits en el procesamiento de la información social, el sesgo de atribución hostil, criterios de evaluación para la toma de decisiones, los esquemas cognitivos y los pensamientos automáticos.

Para Dogde (1986) Crick y Dodge (1994) los déficits interpretativos de la información son un problema que surge durante y a causa de interpretar las señales, debido a la carencia de elementos cognitivos y meta cognitivos 
para juzgar y analizar la realidad social. Tal proceso se establece en cuatro pasos: 1) codificación de información del contexto, 2) representación mental, 3) búsqueda de respuesta, 4) toma de decisión a partir de evaluar las consecuencias y soluciones posibles.

Los pasos muestran el proceso para adquirir los referentes cognitivos y emitir una respuesta. Para ello se acude a la memoria con el objetivo de recapturar información sobre procesos o hechos similares y la forma como se procedió en dicha situación, según las expectativas y la evaluación de consecuencias, y el nivel de profundidad de la experiencia en la memoria, se adopta la respuesta. Los teóricos proponen que, durante el proceso de realizar acciones de pensamiento inmediato, se activan otras estructuras mentales latentes en las cuales se almacena el conocimiento aprendido, siendo estos esquemas determinantes al momento de tomar decisiones ante los hechos sociales.

Pero en la memoria no sólo se almacena información sobre la experiencia y el contexto, sino que aparece una activación emocional. Lemerise y Arsenio (2000) sustentan que, en todo proceso de interacción interpersonal se da una activación emocional, que está enlazada a estructuras mentales latentes que incluyen acontecimientos afectivos. Por lo que, en el proceso de aprendizaje, toda situación de interacción, tiene una naturaleza emocional que incide en su realización, y en especial otros factores que autores como Dogde (2006), han establecido, tales como: la interpretación de señales, el sesgo de atribución hostil, los esquemas cognitivos y los pensamientos automáticos.

Así, en el proceso de aprendizaje surgen como elementos de bloqueo, factores como la agresión reactiva, la cual se desencadena por la percepción de una amenaza o situación hostil, siendo el error determinante el mal procesamiento de información y la interpretación incorrecta de códigos sociales, atribuyendo una intención equivocada a otras personas en una situación sufrida en condiciones similares, y dejando un registro en la memoria. Las investigaciones adelantadas por Dodge (1980) exponen que los alumnos agresivos padecen una tendencia de atribución hostil externa hacia sus compañeros, producto de la interpretación incorrecta de códigos sociales, hecho que lo llevaba a implicarse en conductas de venganza o agresión reactiva en defensa personal Crick y Dodge (1994).

Teóricos como Dodge, McClaskey y Brown (1986) sustentan la existencia significativa entre las tendencias de atribución hostil y la adaptación social 
negativa de los alumnos. Afirman que, los alumnos víctimas desarrollan intenciones agresivas de forma interna, mientras que los agresores, lo hacen de forma externa, es decir, culpando a los otros de la agresión. Esta activación emocional tiene un efecto sobre la interpretación cognitiva, en especial si las emociones son de angustia y ansiedad, y si existe en la historia de vida un esquema disfuncional producto de experiencias tempranas de vida, como abusos físicos, verbales, y psicológicos, o construcción de guiones agresivos incididos por los referentes adultos, los fracasos de superación, o la influencia de un contexto en el que la agresión, la autodefensa o la venganza son aceptados como normales (de Almeida, Santos \& Porto 2016).

Otra de las causas que inciden en el comportamiento de los estudiantes, es el sistema de evaluación de las respuestas y la toma de decisiones, paso crucial, ya que es en este punto donde el estudiante, a partir de su experiencia pasada, toma en consideración la agresión como una alternativa viable conseguir sus propósitos, Crick y Dodge (1994). Dada la importancia de evaluar las respuestas para el comportamiento humano, (Dodge, 2006) intenta explicar su funcionamiento a través de la implementación del modelo "Evaluación de la respuesta y toma de decisiones" (Response Evaluation and Decision, RED), al servir como mediador entre el sesgo de atribución hostil y la respuesta agresiva. Con ello, se esclarecen aspectos relacionados con la impulsividad y la existencia de mecanismos previos que condicionen la respuesta del estudiante.

El aspecto en cuestión, tiene relación con la aparición e incidencia de los esquemas o guiones cognitivos, propuesto por Huesmann (1988). Su planteamiento explica la adquisición y desarrollo de aprendizajes agresivos durante la infancia. La teoría de Huesmann sobre los guiones cognitivos, explican la existencia de procesos de comprensión más allá de los mensajes del texto, su planteamiento propone que la codificación y el recuerdo, se activan por la información almacenada en los guiones. Su precursor fue Frederic Bartlett (1932), profesor británico de psicología experimental de la Universidad de Cambridge, quien se dedicó al estudio del funcionamiento de la memoria en ambientes culturales, y su relación en la vida cotidiana, con relación a la importancia del significado, la reconstrucción y los esquemas. Sus aportes, arrojaron luz sobre la forma como se organiza el conocimiento en la mente de las personas, en sus indagaciones empleaba historias, leyendas y mitos de otras culturas. Autores como Schank (1975), Schank y Abelson (1977), han ampliado la noción de script o guión, que ha llegado a imponerse como versión más representativa de estas ideas. 
Con los aportes de Kintsch y Van Dijk (1978) surge el concepto de dominio, asociado a los referentes conceptuales y los esquemas abstractos que se construyen en la mente debido a la comprensión y producción de discursos. Para Huesmann y Eron (1989), "los guiones son un tipo de esquemas cognitivos establecidos como programas conductuales almacenados en la memoria que se usan como guías de actuación en situaciones sociales y que son aprendidos en la infancia a través de un doble proceso que implica la codificación inicial de comportamientos observados seguida de ensayos repetidos, de tal manera que al principio de su instauración estos guiones son procesos conscientemente controlados y posteriormente, a medida que son usados de forma repetida, se van convirtiendo en procesos automáticos".

El modelo de guiones explica el comportamiento agresivo como producto de guiones aprendidos durante la infancia, los cuales se almacenan en la memoria y se activan ante determinadas situaciones cotidianas. Para Huesmann (1988), si existe un guion en la memoria, es posible que los estudiantes se enfrenten a una situación conflictiva con una respuesta emocional negativa que ya existía en la memoria. Ello determina que todo componente cognitivo al activarse está estructurado a un estado emocional que pervive en el recuerdo, y que está influido por la frecuencia del reforzamiento y por las atribuciones lógicas que se realizan de tales reforzamientos. Lo anterior obedece a que "las influencias del modelamiento y del reforzamiento operan conjuntamente en el aprendizaje social de la agresión en la vida diaria" (Bandura y Robles, 1975, p.319).

Este planteamiento, permite explicar que los estudiantes más agresivos tienen incorporada en su memoria un sistema de creencias normativas que justifican su agresión, a partir de validar estas formas de comportamiento en la solución de problemas o para obtener lo que desea. Hecho que evidencia una conexión directa entre creencias normativas, conciencia moral y la racionalidad práctica para la toma de decisiones, estando presente, tanto en situaciones inesperadas como cotidianas, en las que opera el procesamiento cognitivo automático.

Al respecto, los postulados teóricos de Beck (2003), exponen tres componentes cognitivos presentes en toda acción humana: los esquemas nucleares definidos como "estructuras funcionales de representaciones relativamente duraderas del conocimiento y la experiencia anterior" (Beck y Clark, 1988, p. 382); los esquemas intermedios que inciden en 
la formación de actitudes y creencias sobre el significado de ciertas experiencias que condicionan la interpretación y construcción de la realidad (Sanz y Vázquez, 2008), y los pensamientos voluntarios/ automáticos que son el resultado de procesos cognitivos pasados que ya han sido incorporados.

La teoría de Beck se fija especialmente en los pensamientos automáticos negativos, que, al ser una extensa red de componentes cognitivos, emocionales, motivacionales y conductuales, que inciden en la conciencia y que al no ser cuestionados pueden ser aceptados como verdaderos o correctos. Así, la incorporación de esquemas cognitivos erróneos da como resultado un procesamiento distorsionado de la información, hecho que produce alteraciones en la interpretación de la realidad. Estos pensamientos producen una valoración negativa del otro, motiva los deseos de agresión física o verbal, la percepción de injusticia y amenaza generalizada en el contexto que produce un daño intencionado, deseo de venganza y devolver el daño (Magán, 2010).

En la etapa actual, la formación de los guiones cognitivos, se conciben como factores derivados del racionamiento moral y de la existencia de posibles mecanismos de desconexión moral, sustentados en los déficits o limitaciones en tres niveles de funcionamiento: (a) un déficit en las habilidades sociales, (b) un retraso en el desarrollo del juicio moral y (c) la presencia de distorsiones cognitivas auto-sirvientes (Bandura 1987).

Dentro de los marcos sociales en estudio, se pueden identificar diferentes factores de riesgo que están presentes en el contexto y que inciden en la forma como los estudiantes perciben la realidad del entorno. Entre ellos son, los problemas de la ilegalidad, y los efectos directos que genera en la cultura y los núcleos sociales, además como un carácter determinante en la configuración de los guiones cognitivos tanto agresivos como funcionales, y en su proceso de racionalización para la toma de decisiones prácticas (Pérez, 2014).

\section{Método: Estudio descriptivo - explicativo}

El propósito de la investigación es describir y explicar situaciones y eventos relacionados con la experiencia de aprendizaje de los estudiantes frente a la praxis de las normas en la vida. 
La codificación semántica permitirá evidenciar la existencia de ciertos patrones de comportamiento hallados en los casos de estudio para su posterior análisis. La construcción de conocimiento a partir de las categorías y núcleos de significación semántica, estarán procesadas con Atlas - T, y servirán para elaborar redes semánticas y establecer nuevas inferencias sobre el conocimiento hallado.

\section{Participantes}

Se realizó un grupo focal con estudiantes de bachillerato procedentes del IDC Misael Pastrana Borrero, y el IDC Carlos Pizarro León Gómez. El rango de edad comprendido es entre los 15 y 18 años de edad. El nivel socio-económico de los estudiantes es el estrato dos, su participación fue voluntaria, así como el anonimato de las respuestas. Para su abordaje se aplicaron, entrevista semiestructurada con los siguientes ejes de reflexión: a) Núcleo familiar, fragmentación y violencia (Factor I), b) La institución y sus obstáculos (Factor II), c) El barrio y los lazos vecinales (Factor II), d) La experiencia de Infracción Legal (Factor III), e) La Infracción Legal (Factor IV), y un cuestionario complementario basado en la Escala de Conducta Antisocial y Delictiva, Cuestionario de Agresión Proactiva-Reactiva -RPQ"Reactive-Proactive Aggression Questionnaire.

\section{Procedimiento}

La aplicación de los instrumentos se realizó con dos grupos focales, mediante encuesta y entrevista abierta. La duración de la prueba fue de 45 minutos, empleando el uso de los cuestionarios de forma intercalada, identificando a través de la memoria y el recuerdo los guiones cognitivos que tienen los estudiantes.

\section{Análisis de datos}

Para el proceso de análisis se seguirán los principios de la Teoría Fundamentada (Grounded Theory) propuesta por Strauss y Corbin (2002). Las fases del análisis se especifican a continuación:

i) Preparación de archivos de datos brutos: las respuestas de los participantes serán transcritas e informatizadas en ATLAS.ti 7.0. 
ii) Primer análisis de los datos en bruto: cada investigador identificó y definió fragmentos significativos del texto y los fue codificando de manera intuitiva para establecer códigos teóricos deductivos.

iii) Desarrollo de las categorías iniciales (codificación axial): tras finalizar este proceso y como estrategia encaminada a la validez del estudio se contrastaron los sistemas de categorías con otros estudios. Todo este proceso se registra en notas teóricas o descriptivas que son integradas a la hora de crear el modelo resultante del análisis y en la escritura de los resultados.

iv) Creación de un modelo de categorías principales, secundarias y sus propiedades (codificación selectiva): las categorías se reducen e integran en redes conceptuales descriptivas o explicativas, utilizando el método de comparación constante.

\section{Resultados}

Uno de los factores por los cuales se seleccionó el grupo focal, es por la identificación en algunos de ellos del Síndrome con Trastorno Disocial, el cual, según la Asociación Psiquiátrica Americana (APA) y la Organización Mundial de la Salud (OMS) se identifica por presentar las siguientes características en el comportamiento: trastorno desafiante oposicionista (TDO), trastorno por déficit de atención con hiperactividad (TDAH) y trastornos exteriorizados. Además, pueden aparecer otros síntomas tales como: mentir, usar armas, manipular e instrumentalizar las relaciones, los estudios corroboran que a mayor severidad del padecimiento, mayor agresividad, y su permanencia y profundidad incide para que aparezca en la vida adulta.

Una de las correlaciones clínicas más importantes establecidas a partir del Trastorno disocial es el consumo de alcohol y drogas por parte de los jóvenes, evidenciando que sus conductas tienden a estar relacionadas con la impulsividad, agresividad, búsqueda de sensaciones, y narrativas violentas. Las cuales inciden de manera sistémica en los siguientes aspectos: i) temperamento infantil, siendo el más característico el de adquisición de 
patrones oposicionistas, con bajo nivel de adaptación social; ii) factores parentales siendo el más común, una inadecuada relación paterno-filial; iii) factores familiares siendo frecuente que aparezca en hogares numerosos, con poca estabilidad o cohesión; iv) factor socioeconómico; v) los factores comunitarios, sobre todo en comunidades con elevada criminalidad, vi) y finalmente, los factores escolares, manifestándose en un pobre énfasis académico, y cambios en la conducta y disciplina.

El siguiente cuadro síntesis recoge los guiones cognitivos que tienen los estudiantes y que inciden en la forma como perciben la realidad.

Cuadro 1. Síntesis Entrevistas de Guiones Agresivos.

\begin{tabular}{|c|c|}
\hline \multicolumn{2}{|c|}{ A) Núcleo familiar, fragmentación y violencia } \\
\hline $\begin{array}{c}\text { A.1. } \\
\begin{array}{c}\text { Percepción del } \\
\text { crecimiento }\end{array}\end{array}$ & $\begin{array}{c}\text { Entrevisto 1. } \\
\text { "Las peleas, los insultos y la separación de mis padres, comenzaron a afectar mi } \\
\text { y perdí todo interés por las el estudio". Me hundí en un estado depresivo, sólo quería } \\
\text { morirme, desaparecer, irme lejos... }\end{array}$ \\
\hline
\end{tabular}

Fuente. Elaboración propia

En sus guiones cognitivos aparecen como significativas las experiencias de soledad y abandono, las cuales marcan su forma de percibir y entender la vida, siendo está carga emocional tan fuerte que impide adquirir un referente claro sobre los derechos ciudadanos e imposibilita la formación de estructuras racionales (Ferreyra, 2014). Las dificultades se agravan con los vacíos en el aprendizaje, ya que impide que el estudiante desarrolle su capacidad para asociar el concepto con su significado (denotación y connotación) en un sistema semántico propio, claro y ordenado, siendo esta una etapa intermedia reconocida como un estímulo que pertenece a la lengua y que los expertos Ilaman "diccionario ortográfico de entrada".

Su construcción deficitaria causa daños en la estructura deficitaria y en el sistema semántico del estudiante. Whorf (1939) ha expresado vívidamente la relación entre el lenguaje y el pensamiento al indicar que cada lenguaje particular no es meramente un instrumento reproductor para dar sonido a las ideas, sino que más bien es por sí mismo el que da forma a las ideas, el programa y guía de la actividad mental del individuo, de los análisis de sus impresiones, para la síntesis de aquello que interpreta y piensa. La percepción que tienen los estudiantes sobre su crecimiento, y los factores que aparecen relacionados en sus guiones cognitivos, son un 
factor que obstaculiza el desarrollo cognitivo, generando una limitación en la competencia personal, incidiendo en las capacidades y conductas necesarias para adaptarse con éxito a su entorno social.

Cuadro 2. Síntesis Entrevistas de Guiones Agresivos.

\begin{tabular}{|c|c|c|}
\hline \multicolumn{2}{|c|}{ A) Núcleo familiar, fragmentación y violencia } \\
\hline $\begin{array}{c}\text { A.2 } \\
\text { Experiencias } \\
\text { dolorosas }\end{array}$ & $\begin{array}{c}\text { Entrevistado 2. } \\
\text { "Con mi grupo de amigos, en los tiempos libres nos gustaba caminar por el barrio, } \\
\text { la requisa, un día la forma como un policía me estaba registrando los pantalones, } \\
\text { (con tosquedad), no me gustó, y terminé involucrado en una pelea con el oficial } \\
\text { quien se aprovechó de mí, y terminaron llevándome a patadas al CAl, ya aprendín } \\
\text { que a la autoridad hay que respetarla o le rompen la cara a uno". }\end{array}$ \\
\hline
\end{tabular}

Fuente. Elaboración propia.

En los casos referidos aparecen eventos dolorosos que son significativos por los jóvenes, y hacen alusión a vivencias en los que el aprendizaje se genera de manera violenta. Estas situaciones son sentidas como eventos negativos, que impiden pensar en la incorporación de lógicas de convivencia sustentadas sobre la comprensión desde el respeto por los derechos individuales, en el hogar y en la institución educativa (Pérez, 2013). La afectividad manifestada en las experiencias de crecimiento, lleva a comprender la interconexión entre las dimensiones cognitiva y afectiva, los estudiantes construyen relaciones lógicas y relaciones entre las realidades externas e internas que son el resultado de la dimensión cognitivoafectivas, generando una carga de contenido emocional presente en su relación de aprendizaje con el dolor y con la realidad académica en el aula.

Según Loeber (1998), las familias en las que se usa el maltrato como mecanismo de coacción y castigo para imponer reglas o conseguir la obediencia del hijo, provocan efectos adversos del tipo orden-oposiciónorden, que terminan incentivando actitudes negativas contra la madre. Así, las conductas de la madre maltratadora aparecen en el hijo en conductas como: desobedecer, agredir, y sobre todo en la conducta oposicional. La conducta del hijo provoca castigos más severos, generando un impacto frente a conductas prosociales.

La joven manifiesta que los episodios de depresión la llevaron a cometer un error en la toma de decisiones, hecho que indica que su experiencia no fue bien manejada, tanto por padres como por docentes, siendo este uno de los factores que más incide en la configuración de los guiones cognitivos, 
ya que su cuadro clínico incluye trastornos de atención, apatía, perdida de interés, sentimiento de autoevaluación personal y, pensamientos obsesivos (de la Calle et al., 2014).

La existencia de un déficit atencional general incide en la capacidad limitada del sistema cognitivo, afectando el funcionamiento atencional, debido a que el o la estudiante tiene un estado de ánimo bajo, que genera una relación directa entre los sesgos atencionales, una auto-representación negativa, una pobre estrategia de aprendizaje, dificultad en la toma de decisiones y una alteración en la función ejecutiva. Para Gotlib (2004), los niveles de interferencia e inhibición se relacionan, lo que permite deducir que la inhibición es una respuesta causada por la interferencia.

En el relato, la entrevistada manifiesta que ha experimentado etapas de inestabilidad familiar que inciden en su rendimiento escolar, y en las creencias de que la familia le pueda brindar apoyo y comprensión. Siendo la explicación a este factor, que uno de los resultados con guiones cognitivos afectivos - negativos, ocasiona una mayor interferencia y una mayor inhibición por estados positivos. En este caso, la activación de la vulnerabilidad depende de la predisposición cognitiva hacia los factores precipitantes o activadores, y la facilidad o dificultad del "desenganche" de la situación negativa, lo que provoca consecuencias en el auto concepto y su valoración, así como de la existencia de estrategias de regulación para manejar los síntomas y su impacto en el aprendizaje.

Las contradicciones en los modelos normativos y en los valores de la familia, frente a los modelos normativos de la comunidad, generan una crisis de identidad en el joven que no es fácil de resolver desde sus lógicas, por lo que comienza el tema del ocultamiento, la manipulación y la mentira. Se observa que los guiones cognitivos en los que hay ambigüedad para seguir las normas y las reglas, generan un mayor nivel de confusión que afecta la construcción de la identidad, el desarrollo de la moral autónoma, de la capacidad empática y de la capacidad de autorregulación y autocontrol de su conducta. Así, las asimetrías en las lógicas normativas institución/ familia/comunidad, incide en cuatro dominios: primero, en el desarrollo de la autonomía y el espíritu crítico; segundo, en la internalización de valores como la solidaridad y la camaradería; tercero, en la construcción del juicio moral.

Otro de los factores identificados en los guiones cognitivos de los estudiantes tiene relación con la actitud de los educadores, tanto de 
maestros como de padres, quienes deben de adquirir la capacidad para escuchar, aconsejar y ayudar en la formación del estudiante. Ya que la carencia de material informativo y de sensibilización, sobre temas que contribuyen en la formación de los juicios de razón es fundamental para lograr la autonomía necesaria, y fomentar la confianza en sí mismo para que pueda enfrentar las situaciones dilemáticas y difíciles.

La carencia de un clima escolar y familiar adecuado, que favorezca el intercambio y el diálogo, la confianza y el apoyo mutuo, contribuye en el equilibrio psicológico y afectivo, lo contrario afecta el proceso de adquisición de normas, valores y hábitos de aprendizaje, para resolver las contradicciones de la estructura moral y la resolución de problemas. En el relato de los jóvenes se visualiza que se vieron obligados a comenzar a trabajar siendo niños, fundamentalmente porque su familia los condiciona y ellos sienten la necesidad de convertirse en un aporte económico para el hogar. La existencia de guiones cognitivos en la mente de los estudiantes, basados en problemas de dinero, es una categoría semántica muy fuerte, que impide el interés y la motivación por el aprendizaje.

Para los entrevistados, el espacio de la calle adquiere un carácter habitual, al que están acostumbrados, es un espacio rutinario, que genera un conflicto, ya que se forman tres procesos de socialización, el institucional que impone su modelo normativo, el familiar que se vuelve deficiente y una socialización de la calle.

Con relación al significado y sentido que el barrio y la comunidad adquiere para ellos, se puede señalar que la gran mayoría ha experimentado una socialización en un entorno determinado violento que los jóvenes vivencian desde su niñez más temprana y que incide de manera determinante en la configuración de sus guiones interpretativos de la realidad, en especial frente al tema de la justicia y de la aplicación de las normas en las situaciones de la vida cotidiana.

Según Holyoak (1990) la ambivalencia cognitiva, y la presencia de guiones de impacto como los producidos por la película La Purga, inciden en la construcción del esquema pragmático de razonamiento, sustentado sobre la apropiación de un conjunto de normas y acuerdos necesarios para la actitud prosocial en el estudiante. Según la teoría de los modelos mentales de Johnson-Laird, enfrentar situaciones dilemáticas implica el uso de ciertos modelos de pensamiento, mientras 
que los casos difíciles requieren construir e incorporar diferentes modelos para una acertada toma de decisiones.

Para los jóvenes entrevistados, el entorno donde viven es tranquilo, pero en los guiones cognitivos aparece ya el síndrome del enemigo hostil, donde se genera una percepción negativa del "otro". Entre las causas que fomentan la agresión está el hecho de la frustración por pertenecer a una clase social baja, además de emplear el castigo físico con frecuencia, y el uso de métodos punitivos. Este fenómeno postula que los estudiantes expuestos a una situación hostil de incontrolabilidad, produce un déficit en el aprendizaje de respuestas de éxito.

Las relaciones con el entorno tuvieron en primera instancia una interacción fuerte y se identificaban con él, pero la prevención aparece frente a los amigos y el contexto social, debido a los estereotipos, factor que aumenta la desconfianza en la comunidad y generan prevención con los temas de justicia social, igualdad y/o equidad.

Cuadro 3. Síntesis Entrevistas de Guiones Agresivos.

\begin{tabular}{|c|c|}
\hline \multicolumn{2}{|c|}{ D) La experiencia de infracción legal } \\
\hline $\begin{array}{c}\text { Entrevistado } 2 . \\
\text { D.1. La calle } \\
\text { espacio } \\
\text { sustituto }\end{array}$ & $\begin{array}{r}\text { "El deseo de gastar me llevó un día a tomar dinero sin permiso de mi casa, mi } \\
\text { mamá se dio cuenta y comenzaron los castigos y la desconfianza con mis padres, a } \\
\text { la fecha no he podido volver a recuperarla, y ahora me siento más ofendida, porque } \\
\text { ellos guardan todo con llave". }\end{array}$ \\
\hline
\end{tabular}

Fuente. Elaboración propia.

Los jóvenes en este espacio sustituto (la calle) inician su ingreso a grupos conformados por pares, desarrollando en muchos casos los inicios de conductas infractoras. Para Akers (2006), aprendemos, mantenemos y modificamos la conducta según su relación con otras personas que tenga actitudes favorables a la violación de las normas sociales y jurídicas (asociación/refuerzo diferencial), y cuando la conducta desviada haya sido aprendida y recibido aprobación, o cuando el estudiante esté expuesto a los modelos desviados y realice su conducta por imitación. Según Bandura (año), el aprendizaje vicario es la adquisición de conductas por imitación, incidiendo en procesos como: la atención, la retención, la producción y la motivación. Para Leyens (1994), "mientras la atención y la retención afectan 
esencialmente a la adquisición, la reproducción motriz y la motivación intervienen sobre todo a nivel de rendimiento".

Los entrevistados se mueven en medio de una cultura ilegal que incide en su toma de decisiones, y en especial sobre el aprendizaje, la afectación indirecta del consumo de marihuana entre sus compañeros incide en su nivel de motivación y en la aceptación de las normas y las leyes. Según la teoría del aprendizaje, observar éste tipo de comportamiento está incidido por los motivadores directos y los motivadores vicarios, siendo un factor de riesgo determinante, ya que la motivación vicaria se produce cuando un observador modifica la conducta después de haber observado a otros individuos realizar tal conducta.

El acto de la observación del consumo de marihuana, se convierte en una representación interna que guía la acción conductual; su reforzamiento, va a producir una codificación simbólica, en la cual los procesos de retención se ven afectados por la repetición de los hechos o conductas del modelo observado y por las habilidades cognitivas del observador.

Estas lógicas cultuales generan violencia y reforzamientos de identidades transgresoras, en la generalidad de los casos, y así se confirma en el discurso de este joven. Según Bandura, la construcción de ideas tiene un impacto en los sistemas de representación individual, que se convierten en imágenes y símbolos verbales. Las imágenes pueden ser duraderas o no, según su frecuencia e impacto, mientras que las representaciones verbales, se originan por la codificación del sistema verbal de la realidad observada, y genera una lógica inicial según las características de la interpretación observada. En el contexto social en estudio, la formación de referentes negativos hacia la policía, incide para que los estudiantes desarrollen guiones favorables para su aceptación y para la comprensión de su rol social.

En términos generales los jóvenes han experimentado durante sus años de formación un acercamiento a la cultura delictiva. Se identifica que esta transculturación se asocia a características corporales como lo son el uso de tatuajes, simpatía por los narcos, y pertenecer a grupos sociales marginales. La realidad cognitiva se vuelve expresión en sus cuerpos. Por lo que, con base en su lenguaje, puede mencionar que en los estudiantes predomina un pensamiento concreto y un vocabulario lacónico, empleando la jerga para su codificación grupal. Al respecto refieren que en la actualidad no les gusta emplear códigos verbales porque los estigmatiza. 
Según Berkowitz (1993) la activación de los sistemas cognitivos de representación opera a modo de red, generando un involucramiento de la emoción, los sentimientos, las reacciones expresivo - motoras, y el sistema de pensamientos y recuerdos. Por tal razón, la aparición de un pensamiento adverso, activa la red de emociones en el estudiante, generando diversas reacciones, según el nivel de profundidad y retención en la memoria de los guiones cognitivos.

Cuadro 4. Síntesis Entrevistas de Guiones Agresivos.

\begin{tabular}{|c|c|}
\hline \multicolumn{2}{|c|}{ E) La mirada de una identidad criminógena } \\
\hline E.2. El delito & $\begin{array}{c}\text { Entrevistado 5. } \\
\text { El delito es una forma de ayudarse a salir adelante en la sociedad actual, la } \\
\text { escuela no nos enseña a defender, uno sale y realmente no aprende nada, porque } \\
\text { la educación no tiene una relación significativa con la vida y las expectativas de } \\
\text { nosotros, sino por lo que la sociedad quiere que sea, pero de lo que se trata, es de } \\
\text { tener dinero a costa de algo para sobrevivir, cualquier cosa, por insignificante que } \\
\text { parezca. }\end{array}$ \\
\hline
\end{tabular}

Fuente. Elaboración propia.

En los discursos de los jóvenes entrevistados encontramos que tienen en su gran mayoría claridad respecto a lo que es una infracción a la ley, así queda expresado en sus relatos, pero justifican su actuación ilegal, sobre un supuesto de explotación y aprovechamiento general. Para Berkowitz (1993), son las cogniciones las que determinan de manera relevante el comportamiento. Al ser las interpretaciones el hecho activador, su juicio crítico y valorativo, se relaciona en red de manera semántica y activa la memoria, el sentimiento y las reacciones expresivo - motoras, que pueden inhibir o estimular la conducta agresiva.

Los jóvenes entrevistados refieren que significan el acto de cometer delitos por diversos motivos, entre estos se encuentran las necesidades económicas, la influencia de pares contaminados, sentirse superior a otros, por imitación, para comprar drogas para su consumo, para obtener dinero fácilmente, por gusto y/o costumbre, por no tener acceso a otras oportunidades y para satisfacer necesidades de consumo como vestirse o pasarlo bien. 


\section{Discusión}

La aparición temprana de los guiones cognitivos tiene muchas repercusiones para él o la estudiante en su comportamiento. Su manejo y transformación depende de una política institucional, sustentada desde un enfoque sistémico de las relaciones interpersonales basada en la convivencia, cuyo fin sea la transformación de los pensamientos distorsionados a partir de un currículo educativo especializado. Implica ello, pensar la educación desde las necesidades y problemas cognitivos del estudiante, y de la inserción de dinámicas de mapeo cognitivo y de su transformación, lo cual parte de reconocer la influencia de subsistemas constituidos por los adultos responsables de la actividad escolar, el subsistema de los alumnos y el subsistema de los grupos extra clase.

Según, el informe europeo Proposal for an Action: Plan to Combat Violence in Schools, uno de los objetivos primordiales de las instituciones de educación es erradicar la violencia escolar mediante el uso de instrumentos que logren evitar las conductas antisociales. Establece el informe que toda intención pedagógica debe buscar el bienestar desde la salud mental, y contribuir en mejorar las relaciones interpersonales. Para Ortega (2003) "aprender a convivir es un seguro de habilidades sociales para el presente y para el futuro. Es, por tanto, un indicador de bienestar social. A su vez, visto desde su lado negativo, el efecto del riesgo que supone la permanencia, por tiempo prolongado, en escenarios y sistemas de convivencia muy conflictivos, cuando no claramente violentos, aumenta de forma importante otros riesgos sociales, como la tendencia al consumo de productos nocivos para la salud, hábitos de consumo de tabaco y alcohol, etc.".

Un abordaje sistémico desde el currículo educativo especializado, debe centrar su interés formativo en la construcción de la conciencia moral, el pensamiento democrático, la cooperación y la educación de los sentimientos y las emociones sociales. Su responsabilidad debe recaer sobre los maestros, quienes deben cumplir con el rol de co construir una realidad objetivada, frente a la subjetividad de los estudiantes, desde el cambio de guiones cognitivos negativos.

Para ello, deben identificarse a través de mapeo cognitivo, las conductas disruptivas verbales, las conductas disruptivas motrices, las conductas disruptivas ruidosas, agresivas, y por distracción. Tal intervención debe 
tener un manejo desde la estructura macro curricular, la cual debe ser la estructura didáctica organizativa del currículo, y que debe ser conformada por tres diseños curriculares bien relacionados entre sí: el diseño didáctico macro curricular, el diseño didáctico meso curricular, y el diseño didáctico micro curricular.

Siendo, el diseño curricular el factor fundamental dentro del eslabón del proceso de enseñanza - aprendizaje, ya que debe ser el profesor junto con los estudiantes los que realizan la preparación previa del proceso, como parte de la proyección, pero también estas funciones de dirección se manifiestan durante el desarrollo del mismo, en el que tanto docente como estudiante, deben fomentar un correcto modelo de convivencia, lo cual implica lograr una armonía entre la ley, la moral y la cultura. Para generar cambios en la forma de pensar del estudiante, se debe lograr que el aprendizaje del aula sea significativo y que reemplace la experiencia del aprendizaje extra clase. Implica conseguir en el estudiante el desarrollo de la comprensión del contenido, ya que es el eslabón de la comprensión del contenido el factor pedagógico que le muestra al estudiante el modo de pensar y actuar.

Para caracterizar la apropiación del contenido y el logro del objetivo, en el proceso de enseñanza-aprendizaje se requiere de una caracterización más integral que la que da la asimilación o la profundidad por sí solos. La sistematización se determina por el grado de generalidad de los problemas que puede enfrentar el estudiante al aplicar los contenidos, conocimientos y habilidades. Se establece un orden, en el cual se deben desarrollar las actividades configurantes de los guiones cognitivos, con ello, se busca, incidir en el acto consciente y en la formación semántica de redes neuronales. Estos cambios cerebrales inducidos de manera voluntaria requieren un propósito, un entrenamiento y un esfuerzo, pero un creciente número de estudios que utiliza imágenes cerebrales muestra lo reales que pueden ser esos cambios que se producen en el interior.

Según Begley (2008) "si los descubrimientos de la neuroplasticidad aplicada y auto dirigida llegan a nuestras clínicas, escuelas y hogares, la capacidad de cambiar voluntariamente el cerebro se convertirá en una parte de nuestras vidas y de nuestra comprensión de lo que significa ser humanos". A partir de estos postulados, la intención de modificar las estructuras cerebrales mediante el currículo educativo especializado, se vuelve una tarea factible, que propone el desarrollo progresivo de las estructuras cognitivas en interacción con el medio, de tal forma 
que estimule la construcción y reconstrucción interior de esquemas y representaciones significativas para el estudiante. Para alcanzar tales fines, el modelo educativo concibe la clase no sólo como una unidad horaria, sino una unidad neuropsicológica, en la cual se cumple una actividad de aprendizaje autónoma, auténtica y neuroconfiguradora que tiene una secuencia lógica de momentos, que no necesariamente son lineales, secuenciales o estáticos, ya que, según las necesidades e intereses de los estudiantes, algunos pueden coincidir.

Las etapas de configuración afectiva están compuestas por dos actividades: exploración y motivación. La etapa de configuración instrumental está integrada por dos momentos: construcción de nuevas redes y circuitos neuronales, y la aplicación de sus conocimientos. La etapa de configuración cognitiva está conformada por el momento de evaluación. El momento de exploración, es el momento de recuperación de valores, actitudes, normas de conducta, habilidades, destrezas, conocimientos previos y preconceptos necesarios para el desarrollo de la actividad. Es el momento en que el estudiante está frente a una situación problemática de aprendizaje que despierta su curiosidad y su interés por aprender o descubrir.

En el segundo momento, relacionado con la configuración de nuevas redes y circuitos neuronales, se analiza con los estudiantes las respuestas que ha dado a las actividades programadas y relacionamos con ellos sus conocimientos previos con los contenidos de aprendizaje (reflexión). En este punto tiene lugar el aprendizaje de los nuevos contenidos, desde la memoria inicial hasta la formación de estructuras cognitivas y metacognitivas más complejas, desarrollo del pensamiento, estructura axiológica, configuraciones cerebrales y creación de nuevas redes y circuitos de comunicación neuronal.

Según Begley (2008) "el cerebro es el órgano del comportamiento y el depósito del aprendizaje y la memoria, el cerebro cambia de una manera real y física cuando adquirimos conocimientos nuevos, dominamos una nueva destreza y nos olvidamos de las cosas que han ocurrido". Así, es el docente, quien puede conseguir una modificación en la estructura mental de sus estudiantes, al conseguir cambios a nivel cognitivo, pro-social y afectivo. De ahí que "cuando las neuronas se activan simultáneamente, sus conexiones sinápticas se hacen más fuertes, aumentando la posibilidad de que la activación de una, estimule la activación de la otra (Begley, 2008). 
Así, las operaciones como codificación, recuperación de memoria, almacenamiento en memoria, etc., van a ser determinantes para la configuración de la realidad, ya que la información que proviene del contexto, y que queda mal comprendida, tiene problemas de respuesta en los estudiantes, sobre todo en aquellos que manejan guiones limitados, lo que a su vez genera asociaciones semánticas débiles, con dificultades mayores para el procesamiento de la información. Desde la teoría de Siegler (1996), se valida la importancia y existencia de las estrategias como elemento fundamental en el progreso del rendimiento cognitivo. Implica ello que los proyectos de prevención de la violencia Wald y Kurlander (2003), deben estar concebidos de tal manera que incluyan las normas escolares y las expectativas relacionadas con el comportamiento, las políticas administrativas, las estrategias de seguimiento, el mapeo de guiones cognitivos y pensamientos distorsionados, así como las actitudes y prácticas de los maestros.

Por ser los esquemas estructuras de conocimiento, se vuelven un factor fundamental para interpretar y predecir las experiencias de aprendizaje. Los estudiantes tienen diferentes conocimientos previos en forma de esquemas que deben ser identificados y la tarea del docente, debe ser facilitar información para que se puedan configurar nuevos esquemas que amplíen la experiencia y el marco de interpretación. Ya que son los esquemas activados, los que orientan el interés cognitivo del estudiante hacía la información y su aceptación o rechazo.

Se pueden clasificar tres tipos de aprendizaje: el aprendizaje por agregación, el aprendizaje por ajuste, y el aprendizaje por reestructuración. El aprendizaje por agregación, involucra el uso de esquemas preexistentes para enfrentar una nueva situación, donde el proceso de aprendizaje no modifica el esquema, tan sólo lo activa y el estudiante responde desde la realidad interiorizada. El aprendizaje por ajuste, se da cuando un elemento de aprendizaje incide en la estructura cognitiva y permea información nueva, por lo que el ajuste, implica el cambio de aspectos que forman parte de un esquema a partir de la experiencia de su aplicación, pero sin reestructurarlo por completo (Aparicio \& Ostos, 2017).

Finalmente, está el aprendizaje por reestructuración, tiene como referente una experiencia significativa que lleva a reorganizar los esquemas existentes e incluso generar esquemas nuevos, que permitan facilitar la comprensión de un aspecto nuevo de la realidad, y el cual se puede denominar esquema de conocimiento. Cada persona elabora 
esquemas de conocimiento, y los construye a partir de diferentes sucesos, experiencias, anécdotas personales, actitudes y normas, que sirven de marco interpretativo para la elaboración de sus formas de pensar.

Este fenómeno lo explica Kintsch y Van Dijk (1978) al proponer los modelos de comprensión de textos, para los cuales, afirman, de la existencia de esquemas muy abstractos que guían una percepción general, que según su nivel de alejamiento a las estructuras esquemáticas del texto puede dificultar notablemente el proceso de comprensión. El cual, al no presentarse, es suplantado por los esquemas sociales cognitivos que tienen su origen a partir de la transmisión sociocultural, quien transvasa metas, creencias, actitudes, y roles. Estos esquemas intervienen en el modo y los recuerdos que permanecen en la memoria, y según lo descubierto, el estudiante adopta o acepta, los rasgos que concuerdan con el prototipo son aceptados de manera directa mucho mejor que aquellos que los desafían.

Al respecto Schank y Abelson (1977) sostienen que los esquemas estudiados contienen información sobre las que basamos nuestras predicciones, y sobre las metas que persiguen las personas, siendo un factor fundamental para determinar la comprensión que tenemos del otro y del mundo. Una vez se establecen los guiones cognitivos, las personas presentan problemas para superar las percepciones que tienen de la realidad, pero tal hecho no es imposible, ya que existe una capacidad neuronal para lograrlo.

\section{Conclusión}

La investigación permitió el reconocimiento de guiones cognitivos negativos en los estudiantes causantes del síndrome de atribución hostil, retraso en el desarrollo del juicio moral y la presencia de distorsiones cognitivas auto-sirvientes y de atribución hostil, factores que inciden en el aprendizaje de las reglas, las normas y la incorporación de los principios de la legalidad. Por ser un síndrome cognitivo, tiene hondas implicaciones en el comportamiento y en la capacidad de establecer redes semánticas y neuronales, presentando los estudiantes agresivos las siguientes características: i) realizan un análisis de la situación de acuerdo con sus experiencias pasadas en situaciones similares más que sobre los hechos concretos de la realidad actual; ii) tienen un mayor número de atribuciones 
hostiles, y iii) presentan un déficit en el procesamiento de la información y en la toma de decisiones.

La existencia de déficit cultural, condiciona los procesos de aprendizaje, razón que fundamenta la inserción de un modelo educativo para la prevención del comportamiento delictivo y disocial, mediatizado por la modificación estructural de la cognición a partir de la creación de un neurocurrículo que facilite dicha modificabilidad cognitiva por medio de la mediatización del significado, tanto en el nivel cognitivo como en el nivel afectivo, produciendo a partir del manejo de los input y output informativos, y de una intervención consciente y planificada, desde los factores de resiliencia un mayor nivel de plasticidad cerebral y conducta prosocial.

\section{Referencias bibliográficas}

Aparicio, O.Y., \& Ostos, O.L. (2017). Las TIC como herramientas cognitivas para la investigación. Revista Interamericana de Investigación, Educación y Pedagogía, RIIEP, 10(1). https://orcid.org/0000-0003-3535-6288. https://orcid.org/0000-0002-64779872

Abelson, R.P. (1977). Psychological status of the script concept. American Psychologist, 7, 715-729.

Akers, R. L. (2006). Aplicaciones de los principios del aprendizaje social. Algunos programas de tratamiento y prevención de la delincuencia. En F. Bueno, H. Kury, L. Rodríguez y E.R. Zaffaroni (Eds.). Derecho penal y criminología como fundamento de la política criminal. Madrid: Dykinson.

Bandura, A. (1987a). Pensamiento y acción: Fundamentos sociales. Barcelona: Martínez Roca.

Bandura, A. y Robles E. (1975) Modificación de conducta. Análisis de la agresión y la delincuencia. México: Trillas.

Bandura, A., C. Barbaranelli, G. V. Caprara, \& C. Pastorelli (1996). Mechanisms of moral disengagement in the exercise of moral agency. Journal of Personality and Social Psychology, 71, 364-374.

Bartlett, F.C. (1932). Remembering. A Study in Experimental and Social Psychology. Cambridge: Cambridge University Press. 
Barrantes, H. A., Beltrán, J. E. P., \& Pérez, F. A. R. (2016). Perfil del estudiante de pregrado de la Facultad de Estudios a Distancia de la Universidad Militar Nueva Granada. Revista Interamericana de Investigación, Educación y Pedagogía, RIIEP, 9(2). DOI: https://doi.org/10.22490/25391887.1948

Beck y Clark, (1988). Anxiety and depression: an information processing perspective. Anxiety Research, 1 (1988), pp. 23-36.

Begley, Sh. (2008). Entrena tu mente. Cambia tu cerebro. Editorial Norma. Bogotá.

Berkowitz, L. (1993) Aggression: Its causes, consequences, and control. Temple University Press, Philadelphia.

Crick, N. R., \& Dodge, K. A. (1994). A review and reformulation of social informationprocessing mechanisms in children's social adjustment. Psychological Bulletin, 115, 74-101.

Crick, N. R., \& Dodge, K. A. (1996). Social information-processing mechanisms on reactive and proactive aggression. Child Development, 67, 993-1002.

de Almeida, R. R., Santos, M. F., \& Porto, J. C. (2016). Lectura de textos ficcionales y el enfoque escolar de literatura: contribuciones para una Pedagogía de la Elección. Revista Interamericana de Educación, Pedagogía y Estudios Culturales, 9(1), 35-51. DOI: https://doi.org/10.22490/25391887.1925

de la Calle, C. V., Malaver, M. O., Gallego, J. D. M., Rodríguez, M., Flórez, J. C., Henao, C. E. \& Saldaña, R. (2014). Aportes de los doctorados de educación en ciencia, tecnología y sociedad, desde la sistematización de sus investigaciones doctorales científicas y formativas, 2000-2010. Revista Interamericana de Investigación, Educación y Pedagogía, RIIEP, 7(1). DOI: https://doi.org/10.15332/s1657-107X.2014.0001.04

Dodge, K. A. (2006). Translational science in action: Hostile attributional style and the development of aggressive behavior problems. Development and psychopathology, 18 (2006), pp. 791-814.

Dodge, K. A. (1986). A social information processing model of social competence in children. In M. Perlmutter (Ed.), Minnesota Symposium on Child Psychology (pp. 77125). Hillsdale, NJ: Erlbaum.

Dodge, K. A. (1993). Social-cognitive mechanisms in the development of conduct disorder and depression. Annual Review of Psychology, 44, 559-584.

Dodge, K.A., Pettit, G.S.McClaskey, C. L.\&Brown, M.M. (1986). Social competence in children. Monographs of the Society for Research in Children, 51 (2, núm. De serie 213).

Ferreyra, H. A. (2014). Mesas Socioeducativas para la Inclusión y la Igualdad. Un programa "De todos con todos". Una experiencia en construcción. Revista Interamericana de Investigación, Educación y Pedagogía, RIIEP, 7(2). DOI: https://doi. org/10.15332/s1657-107X.2014.0002.01 
Gibbs, J. C., Potter, G., \& Goldstein, A. P. (1995). The EQUIP program: Teaching youth to think and act responsibly through a peer-helping approach. Champaign, IL: Research Press.

Gotlib, I. H., Krasnoperova, E., Yue, D. N., y Joormann, J. (2004). Attentional biases for negative interpersonal stimuli in clinical depression. Journal of Abnormal Psychology, 113, 121-135. doi:10.1037/0021-843X.113.1.121.

Holyoak, K. J. (1990). Problem solving. En D. N. Osherson y E. E. Smith (Eds.), Thinking: An invitation to cognitive science. Vol 3. Cambridge MA: MIT Press.

Huesmann, L. R., \& Eron, L. D. (1989). Individual differences and the trait of aggression. European Journal of Personality, 3, 95-106.

Huesmann, L.R. (1988). An information processing model for the development of aggression. Aggressive Behavior, 14, 13-24.

Johnson-Laird, P. N. (1983). Mental Models: Towards a Cognitive Science of Language, Inference and Conciousness. Cambridge, M.A.: Harvard University Press.

Kintsch, W. y DIJK, TA. Van (1978): "Toward a model of text comprehension and production". Psychological Review, 85.

Langer, E. (2016). La construcción de confianza para el estudio de prácticas de resistencia en la escolarización de jóvenes en contextos de pobreza urbana. Revista Interamericana de Investigación, Educación y Pedagogía, RIIEP, 9(2). DOI: https://doi. org/10.22490/25391887.1945

Lemerise EA y Arsenio WF (2000). An integrated model of emotion processes and cognition in social information processing. Child Development, 71, 107-118.

Leyens, J., Yzerbyt, V. y Schadron, G. (1994). Stereotypes and social judgeability. Chichester: Wiley.

Loeber, R., Farrington, D.P., StouthamerLoeber, M., and Van Kammen, W.B. (1998). Antisocial Behavior and Mental Health Problems: Explanatory Factors in Childhood and Adolescence. Mahwah, NJ: Lawrence Erlbaum.

Magán, (2010). Factores cognitivos relacionados con la ira y la hostilidad en hipertensión arterial esencial. Universidad Complutense de Madrid, Facultad de Psicología.

Ortega, R.; Del Rey, R. (2003). Investigación Educativa e Intervención Contra la Violencia Escolar en España. El Proyecto Andalucia Antiviolencia Escolar (Andave). Comportamiento Antisocial: Escola e Familia. Coimbra. Ediliber, 2003, 231 - 256.

Pérez, T. H. P. (2013). Aproximaciones al estado de la cuestión de la investigación en educación y derechos humanos. Revista Interamericana de Investigación, Educación y Pedagogía, RIIEP, 6(1). DOI: https://doi.org/10.15332/s1657-107X.2013.0001.05 
Pérez, T. H. (2014). Colombia: de la educación en emergencia hacia una educación para el posconflicto y la paz. Revista Interamericana de Investigación, Educación y Pedagogía, RIIEP, 7(2). DOI: https://doi.org/10.15332/s1657-107X.2014.0002.06

Sanz y Vázquez, (2008). Trastornos del estado de ánimo. Teorías psicológicas 2aa ed. revisada, pp. 271-298.

Schank, R.C. \& Abelson, R.P. (1977). Scripts, plans, goals and understanding. Hillsdale, N. Jersey: Law- rence Erlbaum Ass.

Schank, R.C. "The structure of episodes in memory". En D.G. Bobrow y A. Collins, (1975).

Siegler, R. S. (1996). Emerging minds: The process of change in children's thinking. New York: Oxford University Press.

Strauss, A. L., Corbin, J., \& Zimmerman, E. (2002). Bases de la investigación cualitativa: técnicas y procedimientos para desarrollar la teoría fundamentada (p. 341). Medellín: Universidad de Antioquia.

Tahull, J. (2016). Modernidad, educación y género. El proyecto inacabado. Revista Interamericana de Investigación, Educación y Pedagogía, RIIEP, 9(2), 159-178. DOI: https://doi.org/10.22490/25391887.1947

Wald, J. y M. Kurlaender (2003). "Connected in Seattle An Exploratory Study of Student Perceptions of Discipline and Attachments to Teachers". New Directions For Youth Development. (99) 35-54.

Whorf, Benjamin Lee. (1939). "La relación del pensamiento y el comportamiento habitual con el lenguaje" (1939), en: Lenguaje, pensamiento y realidad. Barcelona: Barral editores, 1971, pp. 155-184. 